\title{
Algunos aspectos clínicos durante la Epidemia de Dengue Hemorrágico en Cuba
}

* Hospital Universitário Pediátrico del Cerro, Havana, Cuba.

**Policlínico Angel Almejeiras, Havana, Cuba.

*** Hospital Pediátrico Willian Soler, Havana, Cuba.

***Hospital Pediátrico Pedro Borras, Havana, Cuba.
* Carlos Dotres Martinez

** Gladys Fallat Machado

* Eric Martinez Torres

Roberto Carpio Sabatela

Eulogio Hernandez

***Manuel Rojo Concepcion

An ou tbreak of hemorrhagic dengue fever occurred in Cuba in 1981. Among a population of about 10 million people, 344.203 persons were affected; $71,3 \%$ of these were actults and the remaining were children. Among the latter the disease was more severe. During the peak of the epidemic up to 11.701 cases were reported in one single day. In this article the organizational aspects of health care delivery durint the epidemics as well as the studies performed in the 2239 patients admited to 3 hospitals of the city of La Habana from June to August 1981 are reported. The most important clinical and laboratory data from sample of 2239 patien ts under 15 years of age are presented. Some therapeutic aspects, including the experience of the author with the use of in tereferon in 166 patients, are also discussed.

\section{"ASPECTOS CLINICOS OBSERVADOS NA EPIDEMIA DE DENGUE HEMORRÁGIC A EM CUBA"}

Uma epidemia de Dengue hemorragica ocorreu em Cuba no ano de 1981. En tre a populaçäo de cerca de 10 milhöes de habitantes, registrarum-se 344.203 casos, sendo $71,3 \%$ em achultos eo restante crianças, ent tre as quais a doença se apresen tou com maior gravidade. Durante o pico da cpidemia, foram notificados 11.271 casos em um único dia. Neste trabalho se expõem os aspectos organizativos do atendimento médico durante a epidemia e o estudo realizado em 2239 pacien tes hospitalizados com diagnóstico confirmado de Dengue hemorrágica, em três hospitais da Cidade de llavana, no periodo junho - agosto de 1981. Apresentam-se as principais manifestaçoes clinicase humorais de uma amostra de 2239 pacientes menores de 15 anos de idade. Discute-se ainda alguns aspectos terapéu ticos, incla sive a experiência do autor com o uso de interferon em 166 pacientes. 
En el año 1981 surge en Cuba un cuadro caracterizado por fiebre, hemorragias, hemoconcentración y shock el cual afectó a miles de personas, produciéndose 158 fallecidos, constituyendo la primera epidemia de Dengue - He. morrágico de las Américas en este Siglo; epidemia causada por el Tipo Il del virus Dengue, la cual fue controlada totalmente, siendo reportado el último enfermo aproximadamente 4 meses después de iniciada*. No se ha reportado en Cuba ningún otro caso de Dengue Hemorrágico después de $-1981^{4}$. Esta epidemia cubana, ha constituido una gran preocupación para los Organismos Internacionales de Salud, ante la posibiliadad de que - la enfermedad se propague a otras zonas de la América donde existan condiciones favorables para su aparición. ${ }^{12}$

\section{Breve hist oria del Dengue en Cuba.}

La primera epidemia de Dengue Clásico en América se inició en las Islas-Vírgenes en 1827 y se extendió posteriormente a Cuba, Venezuela y otros países; con un número considerable de casos en La Habana; entre los años 1885 y 1920 se presentaron muchas epidemias en el Caribe. reportándose - casos en Cuba. ${ }^{6}$ ?

Em 1897 se reportó en La Habana, una epidemia de Dengue con manifestaciones hemorrágicas que coincidió con la reportada ese mismo año em Texas.

En 1945. Pittalogd, citado por Cantelar ${ }^{3}$ publicó impresos sobre un - brote de dengue en La Habana. apare. ciendo por aquel tiempo bortes locales. por la existencia de un limitado número de personas no inmunes.

En 1977 se reporta en Cuba un brote de Dengue producido por el virus I en proporciones epidémicas, reportándose cerca de medio millón de afectados. fundamentalmente en las provincias orientales del país.

En esta epidemia no se registraron casos con manifestaciones hemorrágicas. ${ }^{5}$

\section{El Dengue Hemorrágico en Cuba}

Con el antecedente referido anteriomente durante 1977 y 1978 de haber-se visto afectado el país por uma epidemia de Dengue producido por el - serotipo l y la demostración de títulos elevados de anticuerpos contra - el virus en un $44,46 \%$ de la población cubana *durante el segundo -
* Dirección Nacional de Epide. milogía, Ministério de Salud Pública de la Repúhlica de Cuha.
* Dirección Nacional de Epide. miologia. Ministério de Salud Publica de. la Republica de Cuba. 
trimestre de 1981 comenzaron a aparecer casos, principalmente niños que presentaban un cuadro febril acompañado de hemorragias y shock. Debido - a la falta de experiencia médica en relación a la enfermedad, los primeros casos no fueron diagnosticados.

Con una población cercana a los 10 millones de habitantes, se reportó - que fueron afectados en la epidemia cubana de $1981,344,203$ personas - de los cuales el $71,3 \%$ fueron adultos y el resto niños dentro de los - cuales se presentó con mayor frecuencia y severidad el Dengue Hemorrágico. Durante el mayor día de morbilidad se atendieron 11,721 pacientes.

Se considera que alrededor del $30 \%$ de los enfermos reportados $(110,000)$ pudo haber presentado algunos de los cuatro grados de Dengue Hemorrágico habiéndose habilitado 17,000 camas y se ingresaron 116,143 personas en - cuatro meses desde el 6 de junio al 11 de octubre de 1981. Se presentaron 158 fallecidos, 100 niños y 58 adultos $^{4}$.

Principales aspectos organizativos de la atención médica durante la epidemia de Dengue Hemorrágico em Cuba ${ }^{12}$

Desde el punto de vista organizativo, se consideró esta epidemia como - una emergencia nacional cuyo costo se calcula en decenas de millones de dóllares, tomandose todas las medidas necesarias para garantizar la atención médica de los enfermos así como el control y erradicación del agente trasmisor, el Aedes aegypti.

La epidemia cubana de Dengue Hemorrágico que afectó principalmente a - la población infantil, fue controlada totalmente en cuntro menos lo que demuestra que la Organización de Salud logró combatir con éxito dicha epidemia.

El logro de los objetivos se vió favorecido por la actividad de comisiones asesoras multidisciplinarias, la asesoría internacional y el uso del juicio de expertos.

El diagnóstico precoz de la enfermedad así como las clasificaciones para la atención médica de los pacientes resultaron un instrumento útil - para afrontar las diferentes etapas de la epidemia.

Los aspectos fundamentales que permitieron establecer el diagnóstico - precoz así como la organización durante la epidemia cubana fueron: 
- Existencia de un Sistema Nacional de Balud con cobertura médico preventiva en todo el país y gratuita en todos los niveles de atención.

- Alto nivel científico del grupo de especialistas que aseguraron las - medidas tomadas.

- Creación de un proceder único para todo el país relacionado con el - manejo integral de los pacientes (criterio de ingreso, tratamiento, criterio de alta, seguimiento y otros).

- Habilitación de consultas extras en policlínicos, servicios de urgencias y otras instalaciones para la atención de pacientes.

- Habilitación de todas las camas necesarias en hospitales, centros escolares, campamentos de pioneros y otros para la hospitalización de - todos los pacientes con criterios.

- Existencia al alcance de todos los centros de los medios de diagnóstico necesarios.

- Garantía de toda la sangre y hemoderivados necesarios para el tratamiento de los pacientes.

- Participación de las Organizaciones de Masas y de la comunidad en general durante la epidemia.

Inicialmente se presentaron sobre todo en dos hospitales de Ciudad de La - Habana 364 niños cuyos cuadros clínicos fueron interpretados como Menincoccemia debido al cuadro de shock unido al síndrome purpúrico. Rapidamente nos dimos cuenta que estábamos ante otra entidad ya que en un grupo de población se presentaba un rash febril benigno y en otros el cuadro de shock y sangramien. to; rapidamente se investigó y caracterizó la enfermedad estableciéndose de inmediato las normas y procederes para todo el país.

Considerando individualmente la caracterización clí. nico-humoral de los niños enfermos. se estableció casuísticamente la clasificación de éstos, teniendo en cuenta la conducta a seguir desde la primera consulta en: torio:

Grupo A) Los que conllevariam tratamiento ambula.

Todos los pacientes con dengue clásico sin complica- 
ciones, - sin púrpura ni sangramiento y que no presentaran vómitos.

Grupo B) Los que ingresarían en salas convencionales de hospitales o en camas habilitadas en otras instituciones:

Todos los pacientes con cuadro de dengue clásico y púrpura, sangramientos o vómitos así como los pacientes de los Grados I y II de la clasificación de la OMS. siva:

Grupo C) Los que ingresan en salas de terapia inten-

Serían los pacientes con Dengue Hemorrágico de los grupos III y IV.

Durante la epidemia de Dengue Hemorrágico fueron estudiados en las diversas unidades asistenciales grupos de pacientes tanto desde el punto de vista clínico, humoral. anátomapatológicos, enzimáticos, inmunológico y otros.

Durante la epidemia se hospitalizaron $116,143 \mathrm{pa}$ cientes, es decir el $33.7 \%$ de los enfermos reportados. De los ingresados 10.312 fueron reportados graves y muy graves.

Revisión de algunas características del Dengue Hemorrágico en la epidemia cubana de 1981 en niños hospitalizados de la Ciudad de La Habana. ${ }^{1}, 8,9,10,13$

La red pediátrica hospitalaria en Ciudad de La Habana, está distribuida en 12 hospitales pediatricos para la a tención de medio millón de habitantes menores de 15 años e integrada por 2,500 camas de pediatría. Tres de los hospitales docentes de esta red fueron seleccionados para extraer los datos fundamentales durante la epidemia del Dengue Hemorrágico en 1981 En estos centros se hospita. lizaron 2239 enfermos con el diagnóstico de Dengue Hemorrágico.

Los hospitales señalados son:

- Hospital Pediátrico Docente "William Soler" con 400 camas y 1254 enfermos estudiados.

- Hospital Infantil Docnete "Pedro Borrás" con 300 camas y 202 enfermos estudiados.

- Hospital Pediátrico Docnete de Centro Habana con 260 camas y 783 pacientes estudiados. 
En el período de junio a agosto de 1981 (ambos inclusive), el Hospital Pediátrico Docente "William Soler" atendió en su servicio de urgencia un total de 4699 pacientes entre 0 y 14 años que fueron considerados como enfermos de Dengue. De este total, quedaron hospitalizados 1845 niños $(39,3 \%)$ de los cuales se revisaron los expedientes clínicos de 1254 (67,8\% de los hospitalizados). Hubo 9 fallecidos, que se estudiaron anatomopatológicamente. El total de los pacientes investigados clinicamente cumplía los criterios emitidos por el Comité de Expertos de la OMS para considerarlos portadores FHD/SSD.

\section{Distribución en el tiempo:}

El comportamiento de la morbilidad en la epidemia fue francamente ascendente y rápida en las primeras semanas de junio, se atendieron 81 enfermos en la primera semana, ascendiendo progresivamente a 1248 en la cuarta semana del propio mes. La mayoría de los que consultaron eran escolares o adolescentes $(78,1 \%)$, mientras que los pre escolares $(18,4 \%)$ y lactantes $(3,5 \%)$, constituyeron la minoría. El total de niños atendidos ascendió a 4699.

En el Hospital William Soler fueron hospitalizados en este período 1847 pacientes, la curva de ingresos se com. portó de igual forma que la de morbilidad, la segunda quincena de junio (3era a 6eta semana de la epidemia) fue la época de mayor afluencia de pacientes que necesitaron hospitalización: escolares y adolescentes $79,1 \%$, pre escolares $16,1 \%$ y lactantes $4,8 \%$. La elevación de ingresos a partir de la 8va semana de epidemia, respondió a criteriosa eminentemente epidemiológicos y no a parámetros unicamento clíncio-humorales.

Edad, sexo, raza y estado nutricional:

La mayor incidencia de la enfermedad se produjo en las edades de 5 a 10 años, para el $50 \%$ del total. En otras muestras este grupo de edades ha tendido hasta el $85 \%$ de la incidencia. Si se tiene en cuenta la teoría secuencial, poderíamos explicar la poca presencia de la enfermidad en niños de 0 a 2 años, por la no existencia de anticuerpos heterotípicos al virus dengue.

En relación al sexo no hubo diferecias importantes en cunto a la distribución y un discreto aumento de los pacientes graves en el sexo femenino, no resultó significativo.

El aspecto racial no se incluyó en el estudio del Wil( adernos de Saúde Pública, R.J. 2 (3):148-157, abr/jun, 1987 
liam Soler, ya que las características de heterogenidad y tendencia a la mezcla étnica de nuestra población hace muy difícil valorar resultados al respecto. Sin embargo, fue apreciación general de los médicos del Hospital William Soler que el Dengue Hemorrágico era más grave ne los niños considerados como blancos o europoides. En el Hospital Pediátrico Docente de Centro Habana, Hernández y colaboradores en una muestra de 783 pacientes con Dengue Hemorrágico, encontraron que en todos los grupos de edades predominó el blanco, con $81,9 \%$ de los casos, seguido del mestizo con un $12,9 \%$ y el negro con un $3,6 \%$. Por otra parte en el Hospital Pediátrico Docente Pedro Borrás donde Rojo $^{13}$ y colaboradores estudiaron 202 pacientes, hubo predominio de los classificados como blancos según el color de la piel para un $85,1 \%$, mestizos $9 \%$ y negros $5,9 \%$.

Enr relación al estado nutricional, en los 202 pacientes del Pedro Borrás se observó que el 99,5\% de ellos tenían un estado nutricional adecuado encontrando un solo paciente con desnutrición aguda ligera. En el Pediátrico de Centro Habana, se señala las rarezas de pacientes desnutridos afectados por la forma más grave de Dengue Hemorrá. gico señalándose en esa muestra de 783 enfermos, un 3,3\% de desnutridos, llamando la atención el $20,1 \%$ de sobrepesos.

Algunos aspectos terapeúticos durante la epidemia cubana de Dengue Hemorrágico. ${ }^{1,2,10,11,13,14}$

En línea general en Cuba se utilizó la guía de la OMS en relación a la conducta terapéutica, no obstante conside. ramos realizar algunas observaciones.

El autamiento del dengue no está dirigido actualmen. te a erradicar la causa lo que si se ha logrado en Cuba, donde actualmente los índices de infectación por Aedes aegypti son bajísimos.

El éxito de la terapéutica una vez que se ha presentado la FHD/SSD está relacionado con la precocidad del diagnóstico así como los recursos al alclance del personal médico y paramédico y la capacidad de éste ante los casos presentados y la rapidez del proceso en muchos de ellos, no existiendo tratamiento específico para esta enfermedad, sólo medidas isntomáticas y la terapéutica con coloides, líquid os y sangre según las necesidades de los pacientes.

Dentro de las medidas sintomáticas, generales y específicas en el manejo de pacientes portadores de FHD/SSD, tenemos las siguientes: 
- Hospitalización inmediata del enfermo diagnosticado como FHD/SSD.

- La fiebre alta deberá ser tratada pero nunca con salicilatos por su acción anticoagulante, así como los trastornos que ocasionan en el equilibrio ácido básico. Se recomienda medidas antipiréticas físicas así como el uso de acetaminofen o dipirona.

- Los vómitos que son otro síntoma muy frecuente en la enfermedad y que muchas veces son la la antesala de la hemoconcentración, deben ser tratados precozmente; se recomienda la hidratación oral con jugos de frutas frías o las sales de rehidratación oral utilizadas para el tratamiento de las enfermedades diarréicas. La supresión de la vía oral con hidratación parenteral estaría indicada en caso de vómitos incontrolables. El difenhidrinato, el metilbromuro de homatropina y el hidróxido de aluminio son medicamen. tos que suelen utilizarse como antieméticos y ante la presencia de gastritis frecuentes en la enfermedad.

La hemoconcentración suele presentarse entre el tercer y quinto día de evolución de la enfermedad, generalmente precedida por vómitos, debe ser tratada con hidratación parenteral de solución salina fisiológica, inicialmente a $400 \mathrm{cc} \times \mathrm{m}^{2}$ en $1-2$ horas para después continuar con matenimiento de sol salina al $1 / 2$ a $2000 \mathrm{cc} \mathrm{x} \mathrm{m}{ }^{2}$ durante 4-6 horas más; si alas 8 horas continúa la hemocoon cenración, se recomienda albúmina humana al $10 \%$ a razón de 6 cc $\times \mathrm{Kg}$. si unido a la hemoconcentración hubiese anemia, se recomienda sangre fresca a 10 c.c. $\times \mathrm{Kg}$. Si manifestaciones de sangramiento con trombocitopenia inferior a $\mathbf{3 0 0 0 0}$ plaquetas, plasma rico en plaquetas $10 \mathrm{cc}$. $\mathrm{Kg}$.

- Ante manifestaciones de pré-shock, se recomienda una observación estricta del paciente, con toma sistemática de la T.A. y las mismas medidas terapéuticas planteadas en la hemoconcentración según las variantes.

- Ante las efusiones pleurales, recomendamos el uso de plasma o albúmina con vistas al aumento de la presión oncótica, unido al uso de furosemida para evitar la sobrecarga liquida del espacio vascular y por onde las manifestaciones de sobrehidratación e insuficiencia cardíaca, esta última medida siempre que no exista hemoconcentración.

- Si so presenta shock, so recomienda el tratamiento del mismo en una unidad de terapia aunque no debe remitirse ningún enfermo en este estado. "Las medidas deben 
desarrollarse donde el shock se presente". Inicialmente se recomienda hidratación con Sol. Salina a 400 c.c. $\mathrm{x} \mathrm{m}^{2}$ sup corp. en 1 hora, continuando con $2000 \mathrm{cc}$. $\mathrm{x} \mathrm{m}^{2}$ las próximas 4 horas, si no sale del shock dentro de la primera hora se recomienda el uso de Dextrán de peso medio 10-20 cc. $x \mathrm{Kg}$ deberá tenerse en cuenta corregir la acidosis metabólica y mantener control electrolítico. No deberá utilizarse albúmina en el shock y la sangre y plasma se valorarán según $\mathrm{Hb}$ y coagulación.

- Ante los sangramientos, se recomienda una terapéutica similar a la del pre-shock; teniendo en cuenta la hb., si ésta es menos de $7 \mathrm{gr} \%$ se recomienda uso de sangre fresca y si cuagulopatía plasma fresco con o sin plaquetas en relación a la trombocitopenia. cuado.

- Enc aso de CIV, se recomienda su tratamiento ade-

- Ojo con la sobrehidratación e hiperrelectrolitemia.

- Ojo con el shock reversible.

Fallecidos: ${ }^{8,9,10}$

En el grupo de 2239 pacientes, hubo 11 fallecidos, de los cuales todos presentaron deño hepático dado por ne. crosis (a veces muy extensa), así como degeneración grasa $y$ hemorragia. No hubo signos de hepatitis. No aparecieron partículas virales en el estudio hepático con microscopia electrónica realizada a un fallecido. No se encontró riñón de shock, ni CID u otra afectación visceral atribuible a aquel.

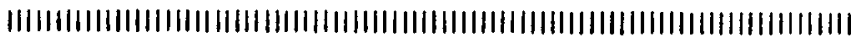

\section{REFERẼNCIAS BIB LIOGRÁFICAS}

1. DOTRLS MARTINL:Z, C. Dengue hemorrígjeo en el niño. In: SI:MINÁRIO DE DI:NGUI: HEMORRÁGICO. Tapachula. 1985. Móxico, OPS, 1985.

2. DOTRLS MARTINLZ, C. et alii. Comportamento clínico humoral y manejo de 166 pacientes portadores de dengue en la ciudad de Pioneros Jusć Marti, Tararí. In: JORNADA NACIONAL DL: PI:DIATRA. 200 Pinar dol Rio. 16.20 dic. 1981. Resumenes. sn.1. p. 34.

3. CANTHLAR, I.N. et alii. Circulación del dengue en Cuba 1978 1979. Re1. Cuh. Med. Trop., 3.3 (1): 72-8. 1981.

4. CASTRO RUZ. I. Discurso por el 28\% aniversário del Asalto al Cuartel Moncada, las Tunas 26 de julio de 1981. Habana, Ld. Pulítica, 1981. 
5. CRUZ PEDROSO, P. Estudio de la epidemia de dengue en el area del Policlinico Docente Pasteur. Habana, 1979. (Tesis)

6 CABIN, A. Declaración sobre el dengue, la fiebre amarella y la erradicación del Aedes aegypti. Bol. OPS, 78 (3): 265-7, 1975.

7. GLSMAN TIRADO, M.V. Dengue: antecedentes históricos, agentes etrológicos, quadro clínico. Rev. Cuh. Med. Trop., 32 (2): $123.30,1980$.

8. HERNÁNDE, E. et alii. Cuadro clínico del dengue en el Hospi. tal Pediátrico Docente de Centro Habana, jun. - sep., 1981. Habana, T.T.R., 1982.

9. LA HOZ, L.M. et alin. Estudio clinico de 292 casos de fiebre hemorrágica dengue. Habana, T.T.R., 1982.

10. MARTINLZ TORRES, E. et alil. Degue hemorrágico en el niño: estudio clínıco patológico. Habana, CNICM, 1984.

11. Nimannitia, S. Dengue haemorrhagic lever. Pediatric Practice, Thailand ia (155-161).

12. RLUNION DHL GRUPO TECNICO ALDES AEGYPTI, DIEN. C;CE Y IIEBRL AMARILLA. Merlda, Yucatan, México, 1-5. jun. 1982 .

13. ROJO, C.M. et aliı. Dengue hemorrígico: estudio clínico de 202 pacientes pedítrcos Fel. Cuh. Pediat., 54 (5):519-38, 1982.

14. WORLD HEALTH ORGANIZATION. Technical Advisory Comittec on Dengue Haemorrhagic lever for the south east Asian and We'stern Pacific Regions Guide for diagnosis. treatment and control of dengue naemorhagic jever. Genevia. 1980. 